Med Klin Intensivmed Notfmed 2014 .

109:478-478

DOI 10.1007/s00063-013-0330-3

Online publiziert: 9. Oktober 2014

(c) Springer-Verlag Berlin Heidelberg 2014

A. Kaltwasser ${ }^{1} \cdot$ C. Dodt ${ }^{2}$

${ }^{1}$ Kreiskliniken Reutlingen $\mathrm{GmbH}$, Reutlingen

2 Notfallzentrum, Städtisches Klinikum München Bogenhausen, München

\title{
Organisationsmodelle der stationären Notfall- und Akutversorgung
} wird. Immer wieder stehen die Teams hier vor besonderen Belastungssituationen, die gemeinsam gelöst werden müssen. aufnahmen häufig kleiner als 50\%. Der größere Anteil wird direkt aus der Notaufnahme wieder nach Hause entlassen, insbesondere wenn viele Notfallpatienten mit Traumata behandelt werden. Notaufnahmen sind somit "Gatekeeper “ für das Krankenhaus und gleichzeitig Schaltzentren für den Versorgungsprozess in den ersten Stunden.

Der Prozess der Notfallversorgung stellt wichtige Weichen für eine erfolgreiche Therapie und ist bei guter Prozessorganisation auch ein ökonomischer Erfolgsfaktor eines Krankenhauses. Intensivstationen als Einheiten zur Therapie schwerstkranker Patienten mit Organversagen sind ein weiterer Bereich, der sich weniger durch eine fachliche Eingrenzung als durch eine spezifische Aufgabenstellung in der Krankenversorgung definiert. In den meisten Krankenhäusern wird diese Aufgabe an Spezialisten für Intensivmedizin delegiert, die bei organspezifischen Problemen durch Fachabteilungen des Hauses unterstützt werden. Auch hier ist eine enge Kooperation der behandelnden Spezialisten ein Schlüssel zum Therapieerfolg.
Notaufnahmen und Intensivstationen ist es gemeinsam, dass in diesen Bereichen, anders als in den Fachabteilungen eines Krankenhauses, ein sehr breites Spektrum von Krankheiten behandelt

\section{Deswegen ist die gute interprofessio- nelle Interaktion besonders wichtig und bedarf großer Aufmerksamkeit.}

Wir wünschen Ihnen eine gewinnbringende Lektüre.

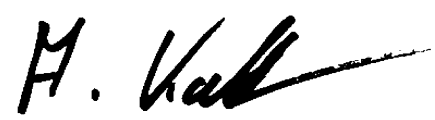

Arnold Kaltwasser
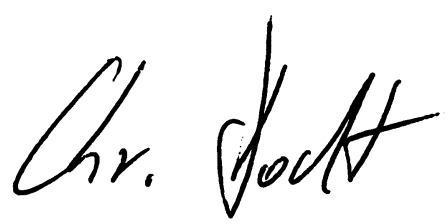

Prof. Dr. Christoph Dodt Zeitschrift Medizinische Klinik - Intensivmedizin und Notfallmedizin wird das auch dadurch unterstrichen, dass seine Herausgeber Vertreter verschiedener Berufsgruppen der Intensivmedizin sind. Neben der interprofessionellen Interaktion ist für die Bereiche Notaufnahme und Intensivstation auch die enge Zusammenarbeit mit den am Haus vorhandenen Fachabteilungen von besonderer Bedeutung. Wie diese Zusammenarbeit in den einzelnen Häusern organisiert wird, ist von den spezifischen Gegebenheiten des jeweiligen Hauses abhängig. In diesem Heft, das sich mit organisatorischen Fragen in der Notfall- und Intensivmedizin beschäftigt, werden unterschiedliche Organisationsmodelle für Intensivstationen, Notaufnahmen und Behandlungseinheiten zur Therapie von Patienten mit Thoraxschmerzen vorgestellt. Zudem wird betrachtet, wie die Organisation eines Schockraums gestaltet und die pflegerische Personalbesetzung einer Intensivstation berechnet werden kann.

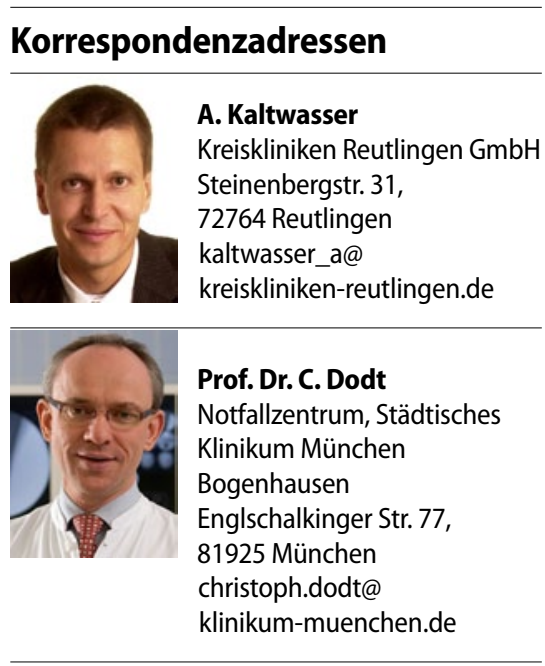

\section{Einhaltung ethischer Richtlinien}

Interessenkonflikt. A. Kaltwasser und C. Dodt geben an, dass kein Interessenkonflikt besteht.

Dieser Beitrag beinhaltet keine Studien an Menschen oder Tieren. 